\title{
THE SCHWARZIAN OPERATOR: SEQUENCES, FIXED POINTS AND $N$-CYCLES
}

\author{
STEPHEN M. ZEMYAN
}

\begin{abstract}
Given a function $f(z)$ that is analytic in a domain $D$, we define the classical Schwarzian derivative $\{f, z\}$ of $f(z)$, and mention some of its most useful analytic properties. We explain how the process of iterating the Schwarzian operator produces a sequence of Schwarzian derivatives, and we illustrate this process with examples. Under a suitable restriction, these sequences become $N$-cycles of Schwarzian derivatives. Some properties of functions belonging to an $N$-cycle are listed. We conclude the article with a collection of related open problems.
\end{abstract}

\section{Definitions}

If $f(z)$ is an analytic, locally univalent (one-to-one) function which is defined in a domain $D$, then the Schwarzian derivative $\{f, z\}$ of $f(z)$ is defined there by

$$
\{f, z\}=\left(\frac{f^{\prime \prime}(z)}{f^{\prime}(z)}\right)^{\prime}-\frac{1}{2}\left(\frac{f^{\prime \prime}(z)}{f^{\prime}(z)}\right)^{2} \text {. }
$$

This definition makes sense, for if $f(z)$ is locally univalent in a neighborhood of $z$, then $f^{\prime}(z) \neq 0$ there. It should be noted that the natural domains of $f(z)$ and $\{f, z\}$ are usually different [1, p. 184]. The Schwarzian operator $\mathcal{S}$ maps $f(z)$ to $\{f, z\}$.

An extremely important and useful property of the Schwarzian derivative is known as the Composition Law. If $f(z)$ is an analytic function and $z=g(\zeta)$ is another analytic function for which the composition $F(\zeta)=f(g(\zeta))$ is defined in some domain, then

$$
\{F, \zeta\}=\{f, g(\zeta)\}\left[g^{\prime}(\zeta)\right]^{2}+\{g, \zeta\}
$$

In particular, if $f(z)$ is analytic in a circular domain centered at the origin, and $|\varepsilon|=1$, then the composition $f_{\varepsilon}(z)=\bar{\varepsilon} f(\varepsilon z)$ is known as a rotation of $f(z)$, and an application of the Composition Law yields the relation $\left\{f_{\varepsilon}, z\right\}=\varepsilon^{2}\{f, \varepsilon z\}$.

In this paper, we investigate the behavior of sequences of Schwarzian derivatives produced by iteration in the following manner. Given a function $f(z)$ that is analytic on the domain $D$, we set $f_{1}(z)=f(z)$, and for $n \geq 1$, we set $f_{n+1}(z)=\mathcal{S} f_{n}(z)$. Each sequence element $f_{n}(z)$ has a natural domain $D_{n}$ of definition, and these domains are usually different. If $f_{N+1}(z)=f_{1}(z)$ on the domain $D_{N+1}=D_{1}$, then the set $\left\{f_{1}(z), \ldots, f_{N}(z)\right\}$ will be called an $N$-cycle of Schwarzian derivatives.

Received by the editors December 9, 2010.

2010 Mathematics Subject Classification. Primary 34L30; Secondary 30D30, 34A25, 34A34.

Key words and phrases. Schwarzian derivative. 


\section{EXAMPLES OF SEQUENCES OF SCHWARZIAN DERIVATIVES}

The functions in each of the following four examples satisfy a functional equation of the form $\mathcal{S} f(z)=\beta f(\alpha z)$. Relations of this form allow for the direct iteration of $\mathcal{S}$.

2.1. Fixed points $(\beta=1$ and $\alpha=1)$. The functions

$$
f(z ; a, b)=\frac{-3 a^{2}}{2(b+a z)^{2}}
$$

are non-trivial fixed points of $\mathcal{S}$, since $\mathcal{S} f(z ; a, b)=f(z ; a, b)$ for any choice of $a \neq 0$ and $b$. Note that $f^{\prime}(z ; a, b) \neq 0$ if $z \neq-b / a$, and that $f(z ; a, b)$ has one double pole at $-b / a$. If $b=0$, then $f(z ; a, 0)=-3 / 2 z^{2}$.

2.2. 2-cycles $(\beta=1$ and $\alpha=-1)$. We can also display a 2-cycle of Schwarzian derivatives. Let

$$
f_{1}(z)=-\frac{4+2 e^{z}+e^{2 z}}{2\left(1+e^{z}\right)^{2}} \quad \text { and } \quad f_{2}(z)=-\frac{1+2 e^{z}+4 e^{2 z}}{2\left(1+e^{z}\right)^{2}} .
$$

Straightforward calculations show that $\mathcal{S} f_{1}(z)=f_{2}(z)=f_{1}(-z)$ and that $\mathcal{S} f_{2}(z)=$ $f_{1}(z)=f_{2}(-z)$. Thus, $\left\{f_{1}, f_{2}\right\}$ is a 2-cycle of Schwarzian derivatives. Since $\mathcal{S}^{2} f_{1}(z)=f_{1}(z)$ and $\mathcal{S}^{2} f_{2}(z)=f_{2}(z)$, both $f_{1}(z)$ and $f_{2}(z)$ can be viewed as fixed points of $\mathcal{S}^{2}$.

The elements of this 2-cycle have some interesting properties:

(1) The sum $f_{1}(z)+f_{2}(z)$ and product $f_{1}(z) f_{2}(z)$ of the cycle elements are even functions.

(2) The zeroes of $f_{1}(z)$ are located at $\ln 2 \pm 2 \pi i / 3 \pm 2 n \pi i$, and the zeroes of $f_{2}(z)$ are symmetrically located at $-\ln 2 \pm 2 \pi i / 3 \pm 2 n \pi i$. Furthermore, all of these zeroes are simple.

(3) The derivatives of the cycle elements,

$$
f_{1}^{\prime}(z)=\frac{3 e^{z}}{\left(1+e^{z}\right)^{3}} \quad \text { and } \quad f_{2}^{\prime}(z)=-\frac{3 e^{2 z}}{\left(1+e^{z}\right)^{3}}
$$

do not vanish.

(4) Since the double poles of both $f_{1}(z)$ and $f_{2}(z)$ are located at $\pm(2 n+1) \pi i$, it follows that $D_{1}=D_{2}$.

This example of a 2-cycle can be generalized. Let

$$
g_{1}(z ; a)=-\frac{4 a^{2}+2 a e^{z}+e^{2 z}}{2\left(a+e^{z}\right)^{2}} \quad \text { and } \quad g_{2}(z ; a)=-\frac{a^{2}+2 a e^{z}+4 e^{2 z}}{2\left(a+e^{z}\right)^{2}} \text {. }
$$

Straightforward calculations show that $\mathcal{S} g_{1}(z ; a)=g_{2}(z ; a)=g_{1}\left(-z ; \frac{1}{a}\right)$ and that $\mathcal{S} g_{2}(z ; a)=g_{1}(z ; a)=g_{2}\left(-z ; \frac{1}{a}\right)$. Thus, $\left\{g_{1}, g_{2}\right\}$ is a 2-cycle of Schwarzian derivatives. Both $g_{1}(z ; a)$ and $g_{2}(z ; a)$ can be viewed as fixed points of $\mathcal{S}^{2}$. The cycle elements $g_{1}(z ; a)$ and $g_{2}(z ; a)$ are translations of $f_{1}(z)$ and $f_{2}(z)$, as

$$
g_{1}(z ; a)=f_{1}(z-\log a) \quad \text { and } \quad g_{2}(z ; a)=f_{2}(z-\log a) .
$$


Consequently, the zeroes and poles of $g_{1}(z ; a)$ and $g_{2}(z ; a)$ are translations of the zeroes and poles of $f_{1}(z)$ and $f_{2}(z)$. Note that if $a=-1$, then both $g_{1}(z ;-1)$ and $g_{2}(z ;-1)$ have double poles at the origin.

2.3. Rational functions of $e^{z}$ with argument doubling $(\beta=4$ and $\alpha=2)$. If

$$
h(z)=-\frac{1+10 e^{z}+e^{2 z}}{8\left(e^{z}-1\right)^{2}}=\frac{5+\cosh z}{8(1-\cosh z)},
$$

then $\mathcal{S} h(z)=\{h, z\}=4 h(2 z)$. Upon iteration, we obtain $\mathcal{S}^{n} h(z)=4^{n} h\left(2^{n} z\right)$. Note that the algebraic form of $h(z)$ is similar to that of $f_{1}(z)$ and $f_{2}(z)$ in the second example above, but that the behavior of the resulting sequence is remarkably different. Note also that $h(z)$ also has a double pole at the origin.

2.4. Elliptic functions with argument doubling $(\beta=-6$ and $\alpha=2)$. The Weierstrass $\wp$-function is the elliptic function defined by

$$
\wp(z)=\frac{1}{z^{2}}+\sum_{\omega}\left(\frac{1}{(z-\omega)^{2}}-\frac{1}{\omega^{2}}\right)
$$

where the sum ranges over all $\omega=n_{1} \omega_{1}+n_{2} \omega_{2}$ except 0 [1, pp. 264-269]. It is well known that $\wp(z)$ satisfies the differential equation

$$
\left(\wp^{\prime}(z)\right)^{2}=4 \wp^{3}(z)-g_{2} \wp(z)-g_{3}
$$

where

$$
g_{2}=60 \sum_{\omega} \frac{1}{\omega^{4}} \quad \text { and } \quad g_{3}=140 \sum_{\omega} \frac{1}{\omega^{6}} .
$$

If we differentiate this differential equation and then divide by $\wp^{\prime}(z)$, we obtain

$$
2 \wp^{\prime \prime}(z)=12 \wp^{2}(z)-g_{2} .
$$

If we differentiate this last equation and then divide by $\wp^{\prime}(z)$, we obtain

$$
\frac{\wp^{\prime \prime \prime}(z)}{\wp^{\prime}(z)}=12 \wp(z) .
$$

It is also well known that $\wp(z)$ satisfies the identity

$$
\frac{1}{4}\left(\frac{\wp^{\prime \prime}(z)}{\wp^{\prime}(z)}\right)^{2}=\wp(2 z)+2 \wp(z) .
$$

By combining the last two equations, we have

$$
\mathcal{S} \wp(z)=\{\wp, z\}=\frac{\wp^{\prime \prime \prime}(z)}{\wp^{\prime}(z)}-\frac{3}{2}\left(\frac{\wp^{\prime \prime}(z)}{\wp^{\prime}(z)}\right)^{2}=-6 \wp(2 z) .
$$

Upon iteration, we obtain $\mathcal{S}^{n} \wp(z)=-6 \cdot 4^{n-1} \wp\left(2^{n} z\right)$. 


\section{TwO THEOREMS CONCERNING $N$-CYCLES}

In this section, we illuminate the structure of $N$-cycles.

Theorem 1. Suppose that $\{f, z\}=f(\alpha z)$ in a circular, $N$-fold symmetric domain $D$ centered at the origin. If $N$ is a positive integer and $\alpha=e^{2 \pi i / N}$, then the set

$$
\left\{\alpha^{2 n-2} f\left(\alpha^{n} z\right)\right\}_{n=1}^{N}
$$

constitutes an $N$-cycle of Schwarzian derivatives. The elements of this $N$-cycle are simply rotations of $f(z)$ multiplied by a suitable power of $\alpha$.

Proof. We first observe that if $\mathcal{S} f(z)=f(\alpha z)$, then

$$
\mathcal{S}^{2} f(z)=\{f, \alpha z\} \cdot \alpha^{2}=\alpha^{2} f\left(\alpha^{2} z\right)
$$

by the Composition Law. By induction, if $\mathcal{S}^{n} f(z)=\alpha^{2 n-2} f\left(\alpha^{n} z\right)$, then

$$
\mathcal{S}^{n+1} f(z)=\alpha^{2 n}\left\{f, \alpha^{n} z\right\}=\alpha^{2 n} f\left(\alpha^{n+1} z\right) .
$$

Now, if $\alpha=e^{2 \pi i / N}$, so that $\alpha^{N}=1$, then $\mathcal{S}^{N+1} f(z)=\mathcal{S}^{1} f(z)=f(\alpha z)$. Thus, the given set constitutes an $N$-cycle of Schwarzian derivatives, as claimed.

Note that $\mathcal{S}^{N} f(z)=f(z) / \alpha^{2}$ belongs to the $N$-cycle. With $|\alpha|=1$, each element of the $N$-cycle is a multiple of a rotation of $f(z)$, since $\alpha^{2 n-2} f\left(\alpha^{n} z\right)=$ $\alpha^{3 n-2} \bar{\alpha}^{n} f\left(\alpha^{n} z\right)=\alpha^{3 n-2} f_{\alpha^{n}}(z)$.

The elements of any $N$-cycle, as described in this theorem, have some very interesting properties:

(1) It is not necessary to apply the Schwarzian operator $N-1$ times to $f(\alpha z)$ in order to compute the rest of the cycle elements; it is only necessary to rotate $f(z)$ sufficiently.

(2) Each element of the $N$-cycle can be viewed as a fixed point of $\mathcal{S}^{N}$, or equivalently, as a solution of the nonlinear ordinary differential equation $\mathcal{S}^{N} f(z)=f(z)$.

(3) If

$$
F_{N}(z)=\sum_{n=1}^{N} \alpha^{2 n-2} f\left(\alpha^{n} z\right)
$$

denotes the sum of the elements of an $N$-cycle, then $F_{N}(z)=\alpha^{2} F_{N}(\alpha z)$. Thus, $F_{N}(z)$ displays $N$-fold symmetry if and only if $N=2$.

(4) If

$$
G_{N}(z)=\prod_{n=1}^{N} \alpha^{2 n-2} f\left(\alpha^{n} z\right),
$$

denotes the product of the elements of an $N$-cycle, then $G_{N}(z)=G_{N}(\alpha z)$. Thus, $G_{N}(z)$ is $N$-fold symmetric for all $N \geq 1$.

(5) The zeroes and poles of any element of the $N$-cycle are exactly the rotations of the zeroes and poles of $f(z)$ or any other cycle element. Thus, every cycle element has the same number of zeroes and poles as any other cycle element.

(6) No cycle element has a pole of order $m \geq 3$. Suppose that $f(z)$ has a pole of order $m \geq 2$ at $z=z_{0}$. Then

$$
\mathcal{S}^{N} f(z)=f(z) / \alpha^{2}=\frac{g(z)}{\left(z-z_{0}\right)^{m}},
$$


where $g(z)$ is analytic at $z=z_{0}$ and $g\left(z_{0}\right) \neq 0$ [1, p. 184]. By direct computation, we obtain the representation

$$
\begin{aligned}
\mathcal{S}^{N+1} f(z) & =\frac{1}{\left(z-z_{0}\right)^{2}}\left[\frac{\left(1-m^{2}\right)}{2}+\frac{\left(m^{2}-1\right) g^{\prime}\left(z_{0}\right)}{m g\left(z_{0}\right)}\left(z-z_{0}\right)+\cdots\right] \\
& =f(\alpha z) .
\end{aligned}
$$

From this representation, it follows that

$$
f(z)=\frac{\alpha^{2}}{\left(z-\alpha z_{0}\right)^{2}}\left[\frac{\left(1-m^{2}\right)}{2}+\frac{\left(m^{2}-1\right) g^{\prime}\left(z_{0}\right)}{\alpha m g\left(z_{0}\right)}\left(z-\alpha z_{0}\right)+\cdots\right] .
$$

To summarize, we have shown that if $f(z)$ has a pole of order $m \geq 2$ at $z=z_{0}$, then $f(z)$ also has a pole of order 2 at $z=\alpha z_{0}$. By repeating this argument $N-1$ more times, we conclude that $f(z)$ has symmetrically placed poles of order 2 at $z=\alpha^{n} z_{0}$ for $n=1, \ldots, N$. In particular, since $z=\alpha^{N} z_{0}=z_{0}$, it must be the case that $m=2$.

(7) The derivative of every cycle element does not vanish. Suppose on the contrary that $f^{\prime}\left(z_{0}\right)=0$. Since $\mathcal{S}^{N} f(z)=f(z) / \alpha^{2}$, it follows from the definition of the Schwarzian derivative that $\mathcal{S}^{N+1} f(z)=f(\alpha z)$ has a pole of order 2 at $z=z_{0}$. By repeating the argument given in the last item, we conclude that $f(z)$ also has a pole of order 2 at $z=z_{0}$, contrary to assumption.

(8) No cycle element has a zero of order $m \geq 2$. Since the proof is entirely similar to the argument above, it is omitted here.

It is easy to state another theorem that characterizes $N$-cycles.

Theorem 2. Suppose that $\{f, z\}=f(z-\log a)$ in some horizontal strip domain $D$. If $N$ is a positive integer and $a=e^{2 \pi i / N}$, then the set

$$
\{f(z-n \log a)\}_{n=1}^{N}
$$

constitutes an $N$-cycle of Schwarzian derivatives.

Proof. If $\mathcal{S} f(z)=f(z-\log a)$, then $\mathcal{S}^{2} f(z)=f(z-2 \log a)$ by the Composition Law. Inductively, $\mathcal{S}^{n} f(z)=f(z-n \log a)$ for all $n$. With $a^{N}=1$, we have $N \log a=0$, so that $\mathcal{S}^{N+1} f(z)=f(z-\log a)=\mathcal{S} f(z)$. Thus, the given set constitutes an $N$-cycle of Schwarzian derivatives, as claimed.

\section{Open PROBLemS}

Some characteristics of cycle elements for all $N$ have been noted above, but their precise form for $N \geq 3$ is an open problem. There are two obvious approaches here.

On the one hand, one could solve the nonlinear ordinary differential equation $\mathcal{S}^{N} f(z)=f(z)$ to determine all $N$ cycle elements at once. After the denominators are cleared in this equation, it becomes a polynomial equation in $f(z)$ and its derivatives whose order and degree depends upon $N$.

On the other hand, one could solve the Schwarzian operator equation $\mathcal{S} f(z)=$ $\{f, z\}=\beta f(\alpha z)$ with $\beta=1$ and $\alpha=e^{2 \pi i / N}$ to determine the one cycle element, and then, according to Theorem 1 , rotate it $N-1$ times to determine the other cycle elements.

Given the need to determine the elements of all $N$-cycles for $N \geq 3$ and the four interesting examples displayed in $\S 2$, it is of interest to solve the Schwarzian operator equation $\{f, z\}=\beta f(\alpha z)$ for all complex values of $\alpha$ and $\beta$. 
It is easy to generalize this problem in many directions. For example, if $P(z)$ denotes an arbitrary polynomial of degree $N$, do functions $f(z)$ exist for which the equation $\{f, z\}=P(f(\alpha z))$ holds on some domain?

\section{REFERENCES}

1. Lars V. Ahlfors, Complex Analysis, McGraw-Hill Book Company, New York, 1966. MR510197 (80c:30001)

Department of Mathematics, Penn State Mont Alto, Mont Alto, Pennsylvania 172379799

E-mail address: smz3@psu.edu 